\title{
BMJ Global Health Accuracy of verbal autopsy, clinical data and minimally invasive autopsy in the evaluation of malaria-specific mortality: an observational study
}

Natalia Rakislova, ${ }^{1,2}$ Dercio Jordao, ${ }^{3}$ Mamudo R Ismail,, ${ }^{4,5}$ Alfredo Mayor, ${ }^{1}$ Pau Cisteró, ${ }^{1}$ Lorena Marimon, ${ }^{1}$ Melania Ferrando, ${ }^{1}$ Juan Carlos Hurtado (1) ,1,6 Lucilia Lovane, ${ }^{4}$ Carla Carrilho, ${ }^{4,5}$ Cesaltina Lorenzoni, ${ }^{4}$ Fabiola Fernandes, ${ }^{4}$ Tacilta Nhampossa, ${ }^{7,8}$ Anelsio Cossa, ${ }^{7}$ Inacio Mandomando (D) ,7 Mireia Navarro, ${ }^{1,6}$ Isaac Casas, ${ }^{1}$ Khatia Munguambe, ${ }^{5,7}$ Maria Maixenchs, ${ }^{1,7}$ Llorenç Quintó, ${ }^{1}$ Eusebio Macete, ${ }^{7}$ Mikel Martinez, ${ }^{1,6}$ Robert W Snow, ${ }^{9,10}$ Quique Bassat (i) , 1,7 Clara Menéndez, ${ }^{1,7,11}$ Jaume Ordi ${ }^{1,2}$

To cite: Rakislova N, Jordao D, Ismail MR, et al. Accuracy of verbal autopsy, clinical data and minimally invasive autopsy in the evaluation of malaria-specific mortality: an observational study. BMJ Global Health 2021;6:e005218. doi:10.1136/ bmjgh-2021-005218

Handling editor Seye Abimbola CM and J0 contributed equally. $\mathrm{CM}$ and $\mathrm{JO}$ are joint senior authors.

Received 21 February 2021 Accepted 12 May 2021

Check for updates

C Author(s) (or their employer(s)) 2022. Re-use permitted under CC BY. Published by BMJ.

For numbered affiliations see end of article.

Correspondence to

Dr Jaume Ordi; jordi@clinic.cat

\section{ABSTRACT}

Background Global malaria mortality estimates are hindered by the low reliability of the verbal autopsy (VA) and the clinical records, the most common sources of information used to estimate malariaspecific mortality. We aimed to determine the accuracy of these tools, as well as of the minimally invasive autopsy (MIA), a needle-based postmortem sampling method, to identify malaria-specific mortality in a large series of deceased patients from Mozambique, using complete autopsy as the gold standard. Methods Observational study that included 264 deaths, occurring at a tertiary level hospital in Mozambique, from 1 November 2013 to 31 March 2015 (17 months-long period). Clinical data were abstracted, a computer coded VA was completed using the clinical data as source of information, and an MIA followed by a complete autopsy were performed. Screening for malaria infection was conducted postmortem to all participants using molecular and histological techniques (PCR and immunohistochemistry).

Findings Malaria infection was considered the cause of death in 6/264 (2.3\%) cases: $2 / 54$ children (3.7\%, both less than 5 years old) and 4/57 (7.0\%) maternal deaths. The sensitivity and specificity of the VA, the clinical data and the MIA to identify malariaspecific deaths were $33.3 \%$ and $96.1 \%, 66.7 \%$ and $96.1 \%$, and $100 \%$ and $100 \%$, respectively. In addition, malaria was identified as a possible contributor in 14 additional patients who died of other diseases. These cases were also accurately identified by the MIA (sensitivity $82.4 \%$, specificity $100 \%$ ).

Interpretation The high sensitivity and specificity of the MIA in identifying malaria may help to improve current estimates of malaria-specific mortality in endemic areas.

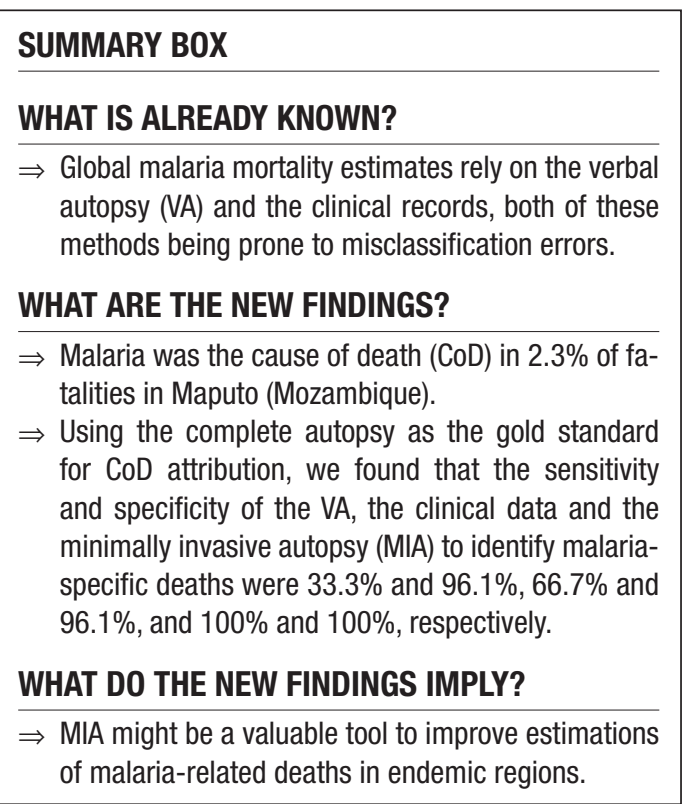

\section{INTRODUCTION}

Cause-specific mortality information is a cornerstone for health policy-making worldwide. ${ }^{1}$ Unfortunately, civil registration systems and vital statistics are poorly functioning in most low-income and middleincome countries, especially in sub-Saharan Africa, where most countries report only fragmentary and sporadic data and where malaria burden is concentrated. ${ }^{23}$ After the launch of the sustainable development goals and the WHO Global Technical Strategy for malaria 2016-2030, ${ }^{45}$ malaria-endemic countries have set as mid-term objective to move towards low transmission or pre-elimination 
status. In this scenario of potentially declining malaria burden, accurate mortality data are crucial to monitor interventions, evaluate progress and adapt strategies. However, obtaining reliable data on malaria-specific mortality, at both national and regional levels, is a major challenge. Indeed, the poor quality and thus reliability of this information seriously hinders the achievement of the Global Technical Strategy goals for malaria 20162030.

Most of the available information on malaria-specific mortality is based on data obtained from clinical records or verbal autopsy (VA). However, both methods have consistently shown limitations and are prone to frequent misclassification errors. ${ }^{6-12}$ Indeed, when clinical diagnoses are contrasted with postmortem findings, clinicopathological discrepancies are frequently reported, particularly in settings with limited availability of diagnostic techniques, ${ }^{13}$ and for infectious diseases. ${ }^{6-9}$ Studies conducted in Malawian children dying with a clinical diagnosis of severe malaria showed significant misdiagnosis in up to a quarter of the cases, where an alternative cause of death $(\mathrm{CoD})$ was identified at autopsy. ${ }^{14}$

The VA tool was developed as an indirect approach to establish the $\mathrm{CoD}$ by interviewing relatives and witnesses of deaths when other registration methods are unavailable. ${ }^{15}$ However, existing VA methods have also serious limitations in attributing deaths to malaria. ${ }^{10}$ An example of the weaknesses of VA was the marked differences around the global malaria mortality estimates produced by the WHO and the Institute of Health Metrics and Evaluation (IHME), ${ }^{11} 12$ with IHME reporting two times as many deaths in the world due to malaria than WHO estimates. More importantly, up to half of the excess malaria deaths were suggested to occur among adults in Africa, ${ }^{12}$ a finding difficult to credit for most malaria experts. Indeed, it is widely accepted that most malariarelated deaths in stable endemic areas are concentrated in children younger than 5 years, ${ }^{10}{ }^{16}$ but the contribution of malaria to mortality in older age groups is still unclear. $^{11} 17$

In the last few years, our group has developed a minimally invasive autopsy (MIA) approach, designed to determine the CoD mainly in low-resource settings, as a feasible alternative to the complete diagnostic autopsy (CDA). ${ }^{18} 19$ The procedure leaves hardly any visible trace on the body, and thus, is more acceptable than the $\mathrm{CDA} .{ }^{20}$ Furthermore, the MIA can be performed by trained technicians, and can be conducted close to the place where death occurs. ${ }^{21}$ Therefore, this approach could be implemented for mortality surveillance, or as a method to complement information gathered through the VA. ${ }^{22}$ The MIA procedure has been validated in perinatal, paediatric, maternal and other adult deaths in Mozambique and Brazil, ${ }^{23-27}$ showing a moderate to substantial concordance with the CDA, particularly for infectious diseases even when evaluated blindly to any additional data, ${ }^{23-26}$ with such concordance increasing to almost perfect when the clinical information is added. ${ }^{28}$
In the present study, we compare the accuracy of a computerised VA, the clinical records, and the MIA to identify malaria-specific deaths in a large series of in-hospital deaths from Mozambique, comparing the results of these methods against the CDA, the gold standard methodology for CoD attribution.

\section{METHODS}

\section{Study area}

The study was conducted at the Maputo Central Hospital (Maputo, Mozambique), a government-funded tertiarylevel hospital, which serves as the referral centre for other hospitals in Southern Mozambique. Maputo urban area has low malaria transmission, although some of the peripheral suburbs and surrounding peri-urban and rural areas have moderately stable transmission. Malaria incidence is higher during the rainy season (October to May), and lower during the dry season (June to September). ${ }^{29}$

\section{Patients included in the study}

During the study period (November 2013 to March 2015) 10.296 deaths occurred at the Maputo Central Hospital. All patients included in this study fulfilled the following criteria: (1) a complete autopsy requested by the in-charge clinician and (2) informed consent to perform the autopsy given by the relatives. In order to avoid selection bias, the first two bodies among all deaths eligible for complete autopsy, closest in time of occurrence to 08:00 am were recruited each morning for the study. Thus, 264 of these deaths (2.5\% of the total deaths) were included in the study to validate the MIA against the CDA. It comprised 41 neonates, 54 children 1 month to 15 years ( 31 children under 5 years), 57 maternal deaths (fulfilling the standard definition of the WHO, ie, death during pregnancy or within 42 days of termination of pregnancy irrespective of its cause) $)^{30}$ and 112 other adults, including 57 men and 55 non-pregnant women. In all cases an MIA was performed, followed by a CDA on the same body. The general characteristics of each group of patients have been reported elsewhere. ${ }^{23-26}$

\section{Patient and public involvement}

No patients or the public were involved in the study design, recruitment, setting the research questions, interpretation or writing up of results, or reporting of the research.

\section{Review of the clinical charts and clinical diagnosis}

The clinical data from all patients were reviewed and abstracted using a standardised questionnaire. A detailed revision of the entire medical record was conducted with abstraction of demographic data, medical history, admission and hospitalisation files, including signs and symptoms, physical examination, laboratory results, imaging reports and treatments received. All clinical diagnoses registered in the medical record by the clinicians in charge prior to death were included in the list 
of clinical diagnoses. Although it was assumed that the first diagnosis listed in the clinical record was the principal diagnosis, we considered malaria as a relevant clinical diagnosis, independently of the order in which it was registered.

A specific search for the malaria diagnostic methods conducted during admission (blood smear and/or rapid diagnostic tests) was performed as part of the review of the clinical charts.

\section{VA diagnosis}

We used the InterVA V.4.04 probabilistic model, one of the most commonly implemented VA tools, ${ }^{31}$ which has a good level of agreement with the physician-coded VA, and its interpretation is reproducible and standardised. ${ }^{323}$ The InterVA calculates the probability of a set of causes of death given the presence of indicators reported in VA interviews. ${ }^{34}{ }^{35}$ In this analysis, the information feeding the model was obtained from the clinical record of the patient and from the obstetric record in perinatal deaths, unified into the WHO 2012 VA standard format, ${ }^{36}$ converted into the 245 input indicators of the VA model. We set malaria prevalence to 'low', and HIV prevalence to 'high' using the InterVA4 package V.1.7.5 implemented in R V.3.5.0 software. ${ }^{37}$

\section{MIA procedure, analysis and diagnosis}

Detailed pathological and microbiological methods of the MIA have been reported elsewhere. ${ }^{19}$ The procedure included disinfection of the surface of the body, followed by the collection of blood and cerebrospinal fluid (CSF) and sampling of key solid organs (liver, lungs, central nervous system (CNS), heart, spleen and kidneys), which were analysed through microbiological and pathological techniques.

The histological evaluation included H\&E stain in all samples and histochemical and/or immunohistochemical stains whenever required to reach a diagnosis. Microbiological methods, which have been reported in detail elsewhere,${ }^{19}$ included investigation for highly incident pathogens. This comprised universal detection of antibodies against HIV-1 and HIV-2, multiplexed PCR analyses for most common respiratory viruses and bacteria, bacterial and fungal cultures using samples of blood, CSF, liver, lungs and CNS; and in some cases, further investigation of bacterial or fungal presence using $16 \mathrm{~S}$ ribosomal RNA (rRNA) gene PCR or the 18S rDNA-ITS PCR, respectively. In patients confirmed to be HIV-positive, an additional microbiological screening was conducted, for common opportunistic pathogens. ${ }^{23-26}$ Other micro-organisms were further investigated depending on the pathological findings observed in the MIA-obtained tissues.

The MIA diagnosis integrated all MIA findings (histology and microbiology), as well as the clinical information.

\section{CDA procedure, analysis and gold standard diagnosis}

Immediately after the MIA, the CDA was performed by another pathologist not involved in the MIA. Histological and microbiological analyses were conducted in samples from the same viscera collected in the MIA and from any grossly identified lesions. The same analytical methods used for the MIA were used for the histological and microbiological samples of the complete autopsy.

The final diagnosis of the CDA integrated all autopsy findings (macroscopy, histology and microbiology), as well as the clinical information and was considered the gold standard for CoD attribution.

\section{Diagnosis of malaria in the MIA and the CDA and definitions}

In all 264 cases, Plasmodium falciparum malaria was proactively screened, and parasite density quantified, using a real-time quantitative PCR (qPCR) assay targeting $18 \mathrm{~S}$ rRNA. Such assay was applied to peripheral blood obtained during the postmortem procedure, and collected as blood spots into Whatman 903 Specimen Collection Paper. ${ }^{38} 39$ Parasitaemia was quantified by extrapolation of cycle thresholds from a standard curve of $P$. falciparum ring-infected erythrocytes.

We selected all the patients with a diagnosis or suspicion of malaria (either clinical, in the VA or in the postmortem examination), and a subset of 10 controls with no suspicion of malaria. In these cases, an immunohistochemical stain was performed in all available tissues with the polyclonal rabbit anti-P. falciparum HPRT1/HPRT antibody (LSBio, Seattle, Washington, USA, $30 \mathrm{~min}$ incubation, dilution 1/1000), and revealed with Envision FLEX and HRP Magenta as chromogen (Agilent, Santa Clara, California, USA) using the Dako PT Link equipment (Agilent). In all these cases a thorough search of malaria parasites was conducted under immersion oil (1000×) both in the H\&E and P. falciparum immunohistochemical stains by two of the investigators (NR and JO). Additionally, a thorough search of haemozoin in macrophages was conducted in all tissues from these patients as evidence of the past malaria.

Malaria was considered the CoD (malaria-specific mortality) based on: (a) presence of cerebral malaria or (b) presence of abundant haemozoin deposition in tissues in the absence of other CoD. Malaria was considered a contributor to death on the basis of: (a) parasitaemia detected by PCR and/or (b) presence of malarial pigment in tissues in the presence of another CoD. In this subset of patients, malaria was considered as a possible contributor to death when haemoglobin levels were below $80 \mathrm{~g} / \mathrm{L}$ (severe anaemia), due to the possible role of malaria in the chain of events leading to death. Malaria was considered as an associated condition death when the haemoglobin levels were within normal limits or showed only mild to moderate anaemia.

\section{Statistical analysis}

The diagnostic performance of the MIA, the clinical records and of the VA to identify malaria as the CoD against the CDA (gold standard) was evaluated as sensitivity, specificity, positive predictive value and negative 
predictive value, and the $95 \%$ CIs for these values were calculated.

Data were analysed with STATA (V.15).

\section{RESULTS}

\section{Malaria-specific mortality}

Malaria was considered the $\mathrm{CoD}$ in 6 out of the 264 autopsies $(2.3 \%)$. Malaria was attributed as the CoD in $2 / 54$ children $(3.7 \%)$, both cases being in children under-five (2/31, $6.4 \%$ of the deaths in children 1 month to 5 years), and in $4 / 57(7.0 \%)$ maternal deaths. No death directly attributed to malaria was identified among neonates or other adults.

The demographic, clinical and laboratory characteristics of the six deaths caused by malaria are shown in table 1. Fever and neurological symptoms were documented in four of the six patients. Of the four maternal deaths one was a primigravid woman and three were multigravida (two gravid 2, one gravid 3). Three deaths occurred in the third trimester immediately after delivery (36, 37 and 38 weeks of gestation). All three women delivered a dead fetus, two after vaginal delivery and one after caesarean section. The fourth malaria maternal death led to a first trimester abortion (8 weeks of gestation). In none of the malaria-related maternal deaths there was a history of pre-eclampsia.

Table 2 shows the histological findings of the six malaria-attributable deaths. In five out of the six cases the diagnosis was based on the histological findings (massive sequestration of parasitised erythrocytes in the CNS, consistent with cerebral malaria). In the sixth patient the diagnosis was reached after clinicopathological correlation: identification of isolated CNS parasites and massive pigment deposition in the spleen and liver plus clinical evidence of severe malaria, in the absence of any alternative CoD.

The immunohistochemical staining revealed, in addition to the parasitised erythrocytes in the CNS capillaries, the presence of the parasitised erythrocytes in the splenic sinusoids in five cases, in the capillaries of the septal alveoli of the lung, in the liver sinusoids in four patients and in the capillaries of the heart and kidney in three cases. Lung oedema was identified in two patients. In one case there was massive sequestration of parasitised erythrocytes in the intestinal capillaries. Most parasitised erythrocytes sequestered in the microvasculature of both the CNS and other viscera were mature stages (trophozoites and schizonts), characterised by the presence of pigment dots in the cytoplasm. Several histological examples of severe malaria cases are shown in figure 1 . No other plausible CoD was identified in any of the six deaths caused by malaria.

No parasites were identified in the immunohistochemical study in any of the 10 cases selected as controls.

\section{Malaria as contributor to death}

Three additional cases tested positive for $P$. falciparum by PCR. One case was a congenital malaria identified in a neonate who showed high blood parasitaemia in the qPCR analysis. Neither parasites, nor malarial pigment were identified in the tissues in the histological and immunohistochemical stains, and a neonatal bacterial sepsis was identified as the CoD. In two cases low blood parasitaemia levels were detected by qPCR (two children aged 7 and 10 years, respectively).

Evidence of past malaria (haemozoin in the liver and the spleen) was identified in further 10 cases (six children and four maternal deaths). No other malaria-related findings were observed, and no parasites were identified in any of these cases through immunohistochemistry. In all these cases a pathologically conclusive $\mathrm{CoD}$ different from malaria was identified.

Table 3 shows the demographic, clinical and laboratory characteristics, as well as the evidence of malaria at the CDA and the final CoD of these 13 patients, who died of other diseases but in whom malaria had possibly contributed to death. Seven of these cases had severe anaemia on the basis of haemoglobin levels below $80 \mathrm{~g} / \mathrm{L}$, and malaria was considered as a significant contributor to death.

\section{MIA diagnosis of malaria}

The histological findings of the MIA of the six deaths caused by malaria are shown in table 2 . The MIA identified all six malaria-specific deaths, and correctly diagnosed all cerebral malaria. Finally, $4 / 7$ cases with malaria as a probable contributor to death and all six cases in which malaria was considered a possible contributor to death were diagnosed by the MIA.

\section{Clinical and VA diagnosis of malaria}

The demographic characteristics, the clinical features, the laboratory data and the final CDA diagnosis of the patients clinically diagnosed of malaria or classified as malaria-specific deaths by the VA are shown in table 4 .

Malaria was clinically diagnosed premortem in 12 patients. In nine of them, malaria was the first clinical diagnosis, whereas in three cases malaria was included in the list of additional diagnoses. Of them, eight patients had received antimalarial drugs. In 11/12 patients the temperature was recorded in the clinical records; 9 of these 11 patients $(82 \%)$ had fever. One case was a child less than 5 years old, two were children aged $6-15$ years, four were maternal deaths and five were other adults. The CDA confirmed a death caused by malaria in four cases whereas in the remaining cases an alternative CoD was identified.

A blood smear for malaria investigation had been performed during admission in 115/264 patients $(43.6 \%)$ and was positive in four cases. A rapid diagnostic test had been performed in 98/264 patients (37.1\%) and was positive in six patients. Two patients had a positive result for both tests (tables 1, 3 and 4). 


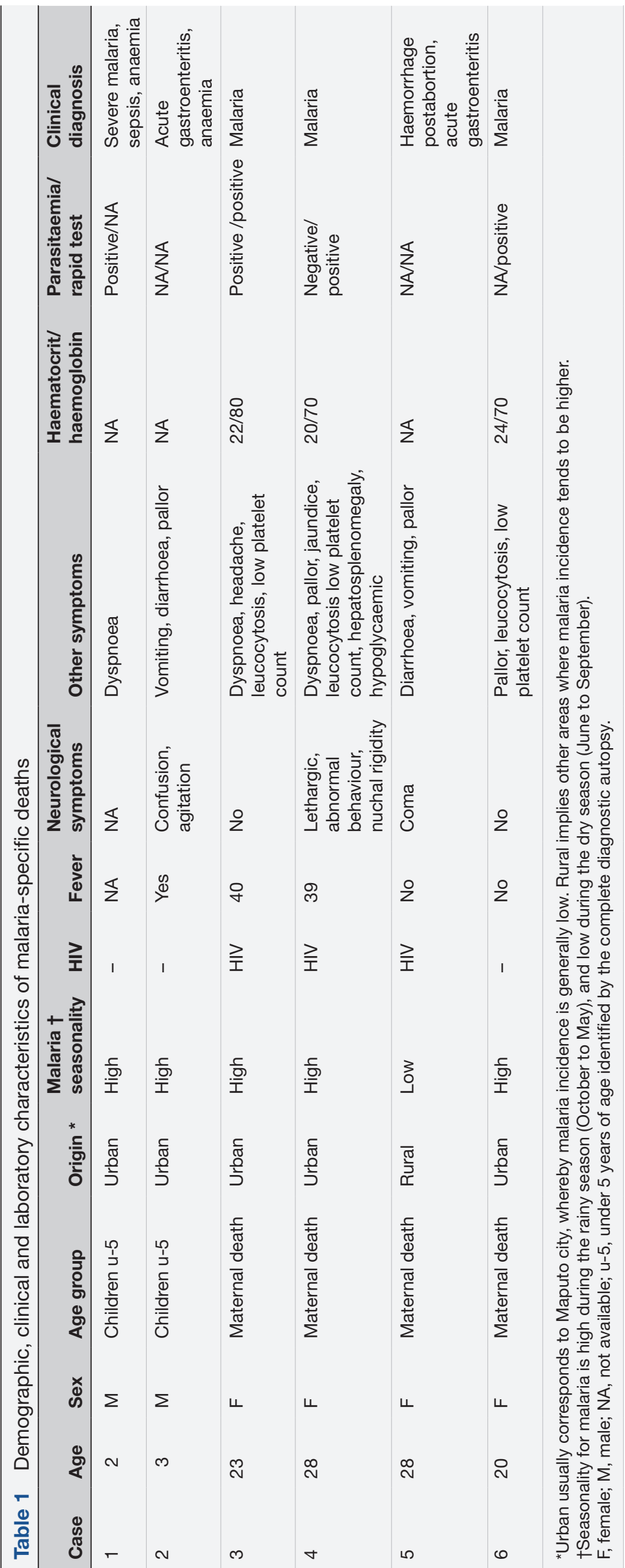

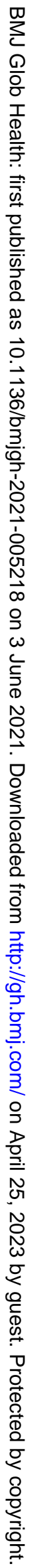




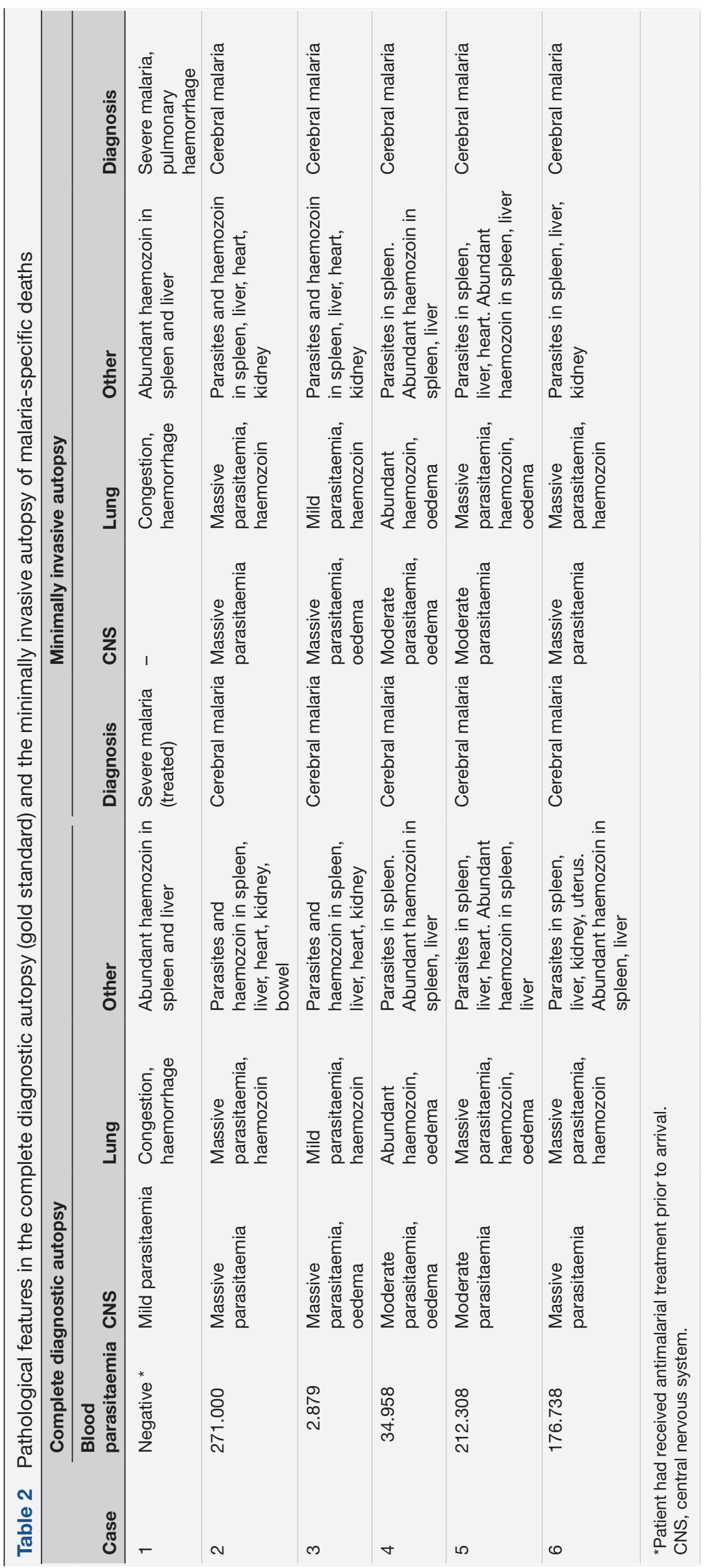

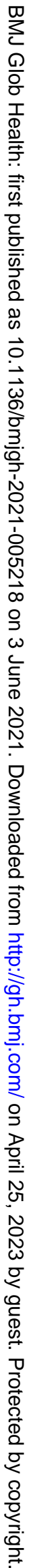




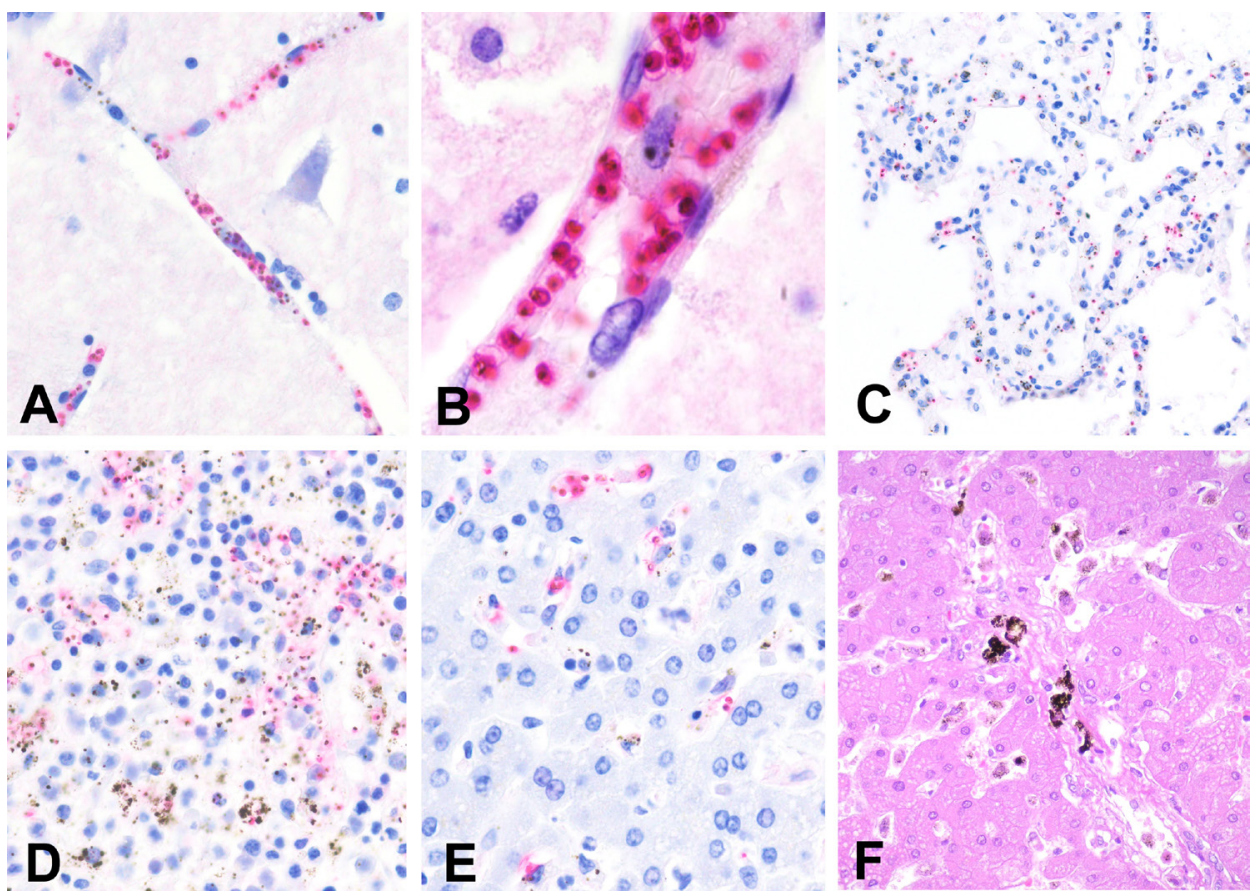

Figure 1 Massive sequestration of Plasmodium falciparum infected erythrocytes in the capillaries of the central nervous system. (A) Cortical vessel showing many parasitised erythrocytes (200x). (B) High power field of single capillaries showing massive parasitised erythrocytes adherent to the endothelium (1000x). Clear haemozoin pigment dots are clearly seen in almost all parasites, indicating that are mature forms. (C) Lung, showing abundant parasitised erythrocytes in the septal capillaries (200x). (D) Spleen showing abundant parasitised erythrocytes in the sinusoids of the red pulp. (E) Liver showing abundant parasitised erythrocytes in the sinusoids (200x). (A-E) Immunohistochemical stain, polyclonal rabbit anti-P. falciparum HPRT1/HPRT antibody. (F) Past malaria with abundant macrophages with malarial pigment in the portal tracts (H\&E, 200x).

Twelve cases were classified as malaria by the VA. In 10 cases, malaria was the only diagnosis provided, whereas in two cases, malaria infection was the second probable CoD. Three cases classified by the VA as malaria-specific deaths were children under-five years of age, four were maternal deaths and five were other adults. The CDA confirmed a death due to malaria in two cases, whereas in the remaining cases an alternative $\mathrm{CoD}$ was identified.

Fever was documented in 97 patients ( $5 / 41$ neonates, 16/31 children under-five, 10/23 children $6-15,17 / 57$ maternal deaths and 49/112 adults). Nine per cent of the febrile deaths were considered clinically and by the VA as deaths caused by malaria $(0 \%$ and $13 \%$, respectively of the febrile deaths occurring in children under-five, $20 \%$ and $0 \%$ of the children $6-15,12 \%$ each or the maternal deaths and $10 \%$ each of the adult deaths). The CDA confirmed malaria-related death in only $3 \%$ of the cases (6\% of the febrile deaths occurring in children underfive and $12 \%$ of the maternal deaths).

\section{Sensitivity and specificity of the clinical records, the VA and the MIA for the diagnosis of malaria as CoD}

Table 5 shows the sensitivity, specificity, positive and negative predictive values of the MIA, the clinical data and the VA in identifying malaria-specific deaths and for all deaths possibly related to malaria, which included the malaria-specific deaths and the deaths in which malaria was a possible contributor (patients with evidence of malaria and severe anaemia). The MIA had a sensitivity and specificity of $100 \%$ to identify malaria-specific deaths. Malaria as a possible contributor to death was identified by the MIA with a sensitivity of $82.4 \%$, and a specificity of $100 \%$. These cases of malaria as a possible contributor were not identified clinically nor by the VA, with the exception of two cases that were mistakenly classified as deaths due to malaria by the clinicians and of two by the VA.

\section{DISCUSSION}

To our knowledge, this is the first time that the MIA method has been compared with the currently used methods to ascertain the CoD (the VA and the clinical records) and shown higher sensitivity and specificity (100\% and $100 \%$, respectively) in identifying malariaspecific mortality. All patients with massive sequestration of $P$. falciparum parasites in the cerebral capillaries, the pathological hallmark of cerebral malaria and the most conclusive evidence of malaria-specific death both in adults and children, were clearly identified by the MIA. Interestingly, whereas $9 \%$ of all febrile deaths were attributed to malaria by the clinicians and the VA, the CDA confirmed malaria-specific death in only $3 \%$ of the patients, with marked discrepancies observed particularly in children other than 5 years and adults, which is in keeping with recent data showing a decrease in the malaria test positivity rate in adults. ${ }^{40}$ Finally, as observed in previous studies, ${ }^{14} 4142$ the sequestration of parasitised 


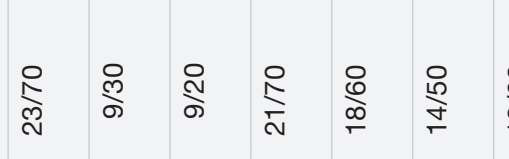

焉主 $\quad 1 \quad 1 \quad$ 主 


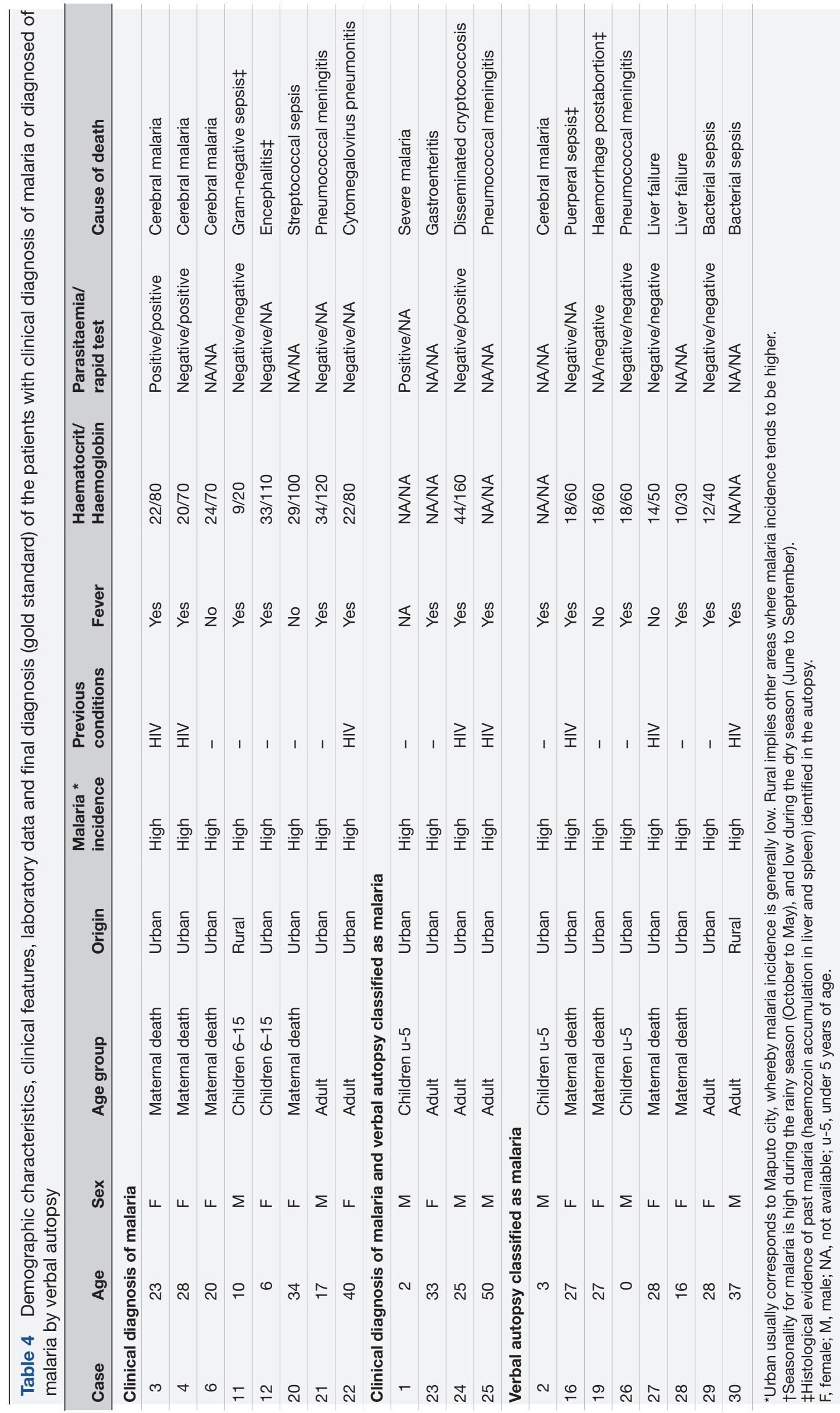


Table 5 Sensitivity, specificity, positive and negative predictive value of the verbal autopsy, clinical and minimally invasive autopsy diagnosis for malaria-specific deaths and for all deaths probably associated with malaria (malaria specific deaths plus patients with any evidence of malaria infection at autopsy and with severe anaemia)

\begin{tabular}{|c|c|c|c|c|}
\hline & Sensitivity & Specificity & Positive predictive value & Negative predictive value \\
\hline \multicolumn{5}{|l|}{ Malaria-specific deaths } \\
\hline Verbal autopsy & $33.3 \%$ (4.3 to 77.7$)$ & $96.1 \%(93.0$ to 98.1$)$ & $16.7 \%(2.1$ to 48.4$)$ & $98.4 \%$ (96.0 to 99.6$)$ \\
\hline Minimally invasive autopsy & $100 \%(54.1$ to 100$)$ & $100 \%(98.6$ to 100$)$ & $100 \%(54.1$ to 100$)$ & $100 \%$ (98.6 to 100$)$ \\
\hline \multicolumn{5}{|c|}{ Deaths probably associated with malaria } \\
\hline Minimally invasive autopsy & $76.9 \%(46.2$ to 95.0$)$ & $100 \%$ (98.5 to 100$)$ & $100 \%(69.2$ to 100$)$ & $98.8 \%$ (96.6 to 99.8$)$ \\
\hline
\end{tabular}

erythrocyte was not restricted to the CNS, but observed in other organs as well.

In this study, two out of the six malaria-specific deaths were clinically missed, yielding a sensitivity of only $66.7 \%$ for the clinical data to ascertain this infection as the CoD. Additionally, the clinicians incorrectly diagnosed 10 cases as malaria. In these 10 cases other causes of death were identified in the CDA (and by the MIA), and no evidence of malaria was found (specificity of $96.1 \%$ ). The challenges of accurately diagnosing infectious diseases in low resource settings have been highlighted in several studies, with increasing frequency of clinical errors when ancillary diagnostic tests are limited, ${ }^{6-9}$ a frequent situation in low-income and middle-income countries, including malaria-endemic countries. This study highlights the urgent need of increasing the diagnostic capacity in these settings, which could, not only improve current data on $\mathrm{CoD}$, but more importantly reduce mortality.

The InterVA model missed four of the six malariaspecific deaths (sensitivity of $33.3 \%$ ). Remarkably, although the InterVA model correctly identified the two paediatric cases, it missed out all maternal deaths caused by malaria, indicating that this VA model has limitations in correctly identifying malaria-specific deaths in this high-risk group. On the other hand, 10 patients classified by the model as malaria-related deaths had alternative causes of death in the CDA and no postmortem evidence of malaria infection. Interestingly, although the VA was fed using clinical data, only four cases were classified as deaths caused by malaria both clinically and by the VA, whereas eight cases not diagnosed clinically as malaria were classified as malaria-specific deaths by the VA. Overall, VA validation studies have shown low levels of sensitivity $(19 \%-75 \%)$ and specificity $(69 \%-100 \%)$ for attributing malaria infection as CoD. ${ }^{43-49}$ Reasons for the low diagnostic performance of the VA in attributing malaria as a $\mathrm{CoD}$ include its poor specificity for diseases with overlapping clinical symptoms, such as fever and neurological or respiratory symptoms. This is the case of malaria infection, meningitis, pneumonia, sepsis, as well as opportunistic HIV-related infections, all of these infections being highly prevalent in many sub-Saharan African countries. ${ }^{50} 51$ Finally, the VA is markedly influenced by the malaria epidemiology, and in endemic areas it tends to assign to malaria any acute febrile illness in the absence of an obvious alternative cause. ${ }^{44}$

In addition to accurately identifying malaria-specific deaths, the MIA captures with relatively high precision patients who were considered to die of other diseases but who had low-level parasitaemia, or evidence of past malaria infection, and in whom malaria may have contributed to death, including patients with severe anaemia in whom malaria was a probable contributor to death (sensitivity $76.9 \%$, specificity $100 \%$ ). Most of these cases were either not identified, or wrongly classified as malariaspecific deaths clinically and by the VA. The difficulties of assigning malaria as an underlying, contributing or indirect CoD have been noted, ${ }^{10}$ and the MIA could provide relevant information in this challenging area. In malariaendemic areas, the progressive acquisition of natural immunity allows the common occurrence of malarial infection without clinical symptoms. Such malaria 'tolerance' is a confounding factor when investigating disease and death, as the detected parasitaemia cannot be ruled out as the aetiology of the disease and death. The MIA may offer an opportunity to improve our knowledge on the indirect contribution of malaria to death.

In this study, malaria-specific deaths occurred only in two groups, namely, children younger than 5 years of age and pregnant women. Indeed, although some reports suggest that cerebral malaria is a common cause of maternal mortality in areas of stable transmission, ${ }^{42} 52$ it is generally considered that malaria-specific maternal deaths are largely restricted to low and unstable malaria transmission areas. ${ }^{17}$ In this regard, studies focusing at determining the real contribution of malaria to maternal mortality are warranted in areas with different malaria transmission. Interestingly, none of the malaria-specific deaths occurred in children aged 6-15 or in adults other than maternal deaths, which is in contrast with some reports suggesting that malaria is responsible for up to $25 \%$ of deaths in children $6-15$ years old and a significant 
number of deaths in adults, ${ }^{15} 5354$ particularly among elderly people. ${ }^{55}$ Moreover, no case of low-level parasitaemia or past malaria was identified among adults, which may be an indirect evidence of the limited contribution of malaria to mortality in this age group.

Our study has several strengths. First, it evaluates the performance of the VA, the clinical records and the MIA against the CDA, the gold standard for CoD attribution. Notably, the CDA included an extensive microbiological testing comprising specific qPCR for malaria infection. Another strength is that all age groups were included in the analysis. The main limitations of the study include a relatively small number of cases, particularly of some age groups, like young children, which may partly explain the small number of malaria-specific deaths. However, the complexity of the CDA and the scarcity of specialised personnel preclude a widespread use of these studies. In addition, the small number of malaria-specific deaths identified in our study may be considered as a limitation. Further studies in high malaria burden settings are warranted to confirm our results. Also, the fact that the study is based in a tertiary hospital might be seen as a limitation to extrapolate findings to deaths occurring in rural health facilities or in the community, since causes of death in rural settings may be different to that of those occurring in a hospital. However, the main objective of this study was evaluating the MIA against a reliable gold standard. Finally, the VA results might have been influenced by the fact that the indicators used to estimate the $\mathrm{CoD}$ by the VA model were extracted from medical records, which may have resulted in some missing indicators. Nevertheless, most of these missing indicators were secondary questions related to the duration of the event. ${ }^{57}$

In conclusion, this study provides evidence on the high sensitivity and specificity of the MIA method in identifying malaria-specific deaths. It also shows that the VA and the clinical records have serious limitations in recognising malaria infection as $\mathrm{CoD}$ in an endemic setting. The MIA, implemented in selected surveillance sites, could be used to help clinicians to reduce diagnostic errors, to improve the quality and performance of current VA tools, to mathematically modulate the data provided by the VA, and ultimately, to improve allcause and disease-specific statistics.

\section{Author affiliations}

${ }^{1}$ ISGLOBAL, Hospital Clinic, University of Barcelona, Barcelona, Spain

${ }^{2}$ Department of Pathology, Hospital Clínic, University of Barcelona, Barcelona, Spain

${ }^{3}$ Department of Pathology, Quelimane Central Hospital, Quelimane, Mozambique

${ }^{4}$ Department of Pathology, Maputo Central Hospital, Maputo, Mozambique

${ }^{5}$ Faculty of Medicine, University Eduardo Mondlane, Maputo, Mozambique

${ }^{6}$ Department of Microbiology, Hospital Clínic, University of Barcelona, Barcelona, Spain

${ }^{7}$ Centro de Investigação em Saúde de Manhiça, Manhica, Mozambique

${ }^{8}$ Department of Pediatrics, Maputo Central Hospital, Maputo, Mozambique

${ }^{9}$ Population and Health Unit, KEMRI - Wellcome Trust Research Programme, Nairobi, Kenya

${ }^{10}$ Centre for Tropical Medicine and Global Health, Nuttfield Department of Clinical Medicine, University of Oxford, Oxford, UK
${ }^{11}$ Consorcio de Investigación Biomédica en Red de Epidemiología y Salud Pública (CIBERESP), Madrid, Spain

Acknowledgements We would like to thank the families of the deceased patients included in this study. The authors are grateful to all the members of the Department of Pathology of the Maputo Central Hospital, whose support made this study possible and also to the staff of the 'Centro de Investigação em Saúde de Manhiça' for their logistic support. We specifically thank Mr Bento Nhancale for his invaluable support to the study.

Funding Funded by the Bill \& Melinda Gates Foundation (Global Health grant numbers OPP1067522 and OPP1128001), and by the Spanish Instituto de Salud Carlos III (FIS, PI12/00757; CM). CISM is supported by the Government of Mozambique and the Spanish Agency for International Development (AECID). RWS is supported by the Wellcome Trust as a Principal Fellow (\#212176) and acknowledges the support of the Wellcome Trust to the Kenya Major Overseas Programme (\# 203077).

\section{Competing interests None declared.}

\section{Patient consent for publication Not required.}

Ethics approval The study received the approval of the Clinical Research Ethics Committee of the Hospital Clinic of Barcelona (Spain; approved, File 2013/8677) and the National Bioethics Committee of Mozambique (342/CNBS/13). As per Ethics Committee local recommendations, MIA and complete autopsy procedures were only conducted after verbal informed consent was provided by the relatives.

Provenance and peer review Not commissioned; externally peer reviewed.

Data availability statement All data relevant to the study are included in the article.

Open access This is an open access article distributed in accordance with the Creative Commons Attribution 4.0 Unported (CC BY 4.0) license, which permits others to copy, redistribute, remix, transform and build upon this work for any purpose, provided the original work is properly cited, a link to the licence is given, and indication of whether changes were made. See: https://creativecommons.org/ licenses/by/4.0/.

\section{ORCID iDs}

Juan Carlos Hurtado http://orcid.org/0000-0001-9519-6082

Inacio Mandomando http://orcid.org/0000-0002-1078-2187

Quique Bassat http://orcid.org/0000-0003-0875-7596

\section{REFERENCES}

1 Black RE, Morris SS, Bryce J. Where and why are 10 million children dying every year? Lancet 2003;361:2226-34.

2 Mikkelsen L, Phillips DE, AbouZahr C, et al. A global assessment of civil registration and vital statistics systems: monitoring data quality and progress. Lancet 2015;386:1395-406.

3 Gelband H, Bogoch II, Rodriguez PS, et al. Is malaria an important cause of death among adults? Am J Trop Med Hyg 2020;103:41-7.

4 WHO. Global technical strategy for malaria 2016-2030, 2016. Available: https://www.who.int/malaria/publications/atoz/ 9789241564991/en/ [Accessed 27 May 2020].

5 United Nations. Resolution adopted by the general assembly on 25 September 2015. Transforming our world: the 2030 agenda for sustainable development; 2015

6 Cox JA, Lukande RL, Lucas S, et al. Autopsy causes of death in HIV-positive individuals in sub-Saharan Africa and correlation with clinical diagnoses. AIDS Rev 2010;12:183-94.

7 Murray J, Sonnenberg P, Nelson G, et al. Cause of death and presence of respiratory disease at autopsy in an HIV-1 seroconversion cohort of southern African gold miners. AIDS 2007;21 Suppl 6:S97-104.

8 Ordi J, Ismail MR, Carrilho C, et al. Clinico-Pathological discrepancies in the diagnosis of causes of maternal death in subSaharan Africa: retrospective analysis. PLoS Med 2009;6:e1000036.

9 Ordi J, Castillo P, Garcia-Basteiro AL, et al. Clinico-Pathological discrepancies in the diagnosis of causes of death in adults in Mozambique: a retrospective observational study. PLoS One 2019;14:e0220657.

10 Herrera S, Enuameh Y, Adjei G. A systematic review and synthesis of the strengths and limitations of measuring malaria mortality through verbal autopsy a systematic review and synthesis of the strengths and limitations of measuring malaria mortality through verbal autopsy. Malar J 2017;16:421. 
11 World Health. Organization (WHO). World malaria report, 2011. Available: https://www.who.int/malaria/world_malaria_report_2011/ en/ [Accessed May 28, 2020].

12 Murray CJL, Rosenfeld LC, Lim SS, et al. Global malaria mortality between 1980 and 2010: a systematic analysis. Lancet 2012;379:413-31.

13 Schwanda-Burger S, Moch H, Muntwyler J, et al. Diagnostic errors in the new millennium: a follow-up autopsy study. Mod Pathol 2012;25:777-83.

14 Taylor TE, Fu WJ, Carr RA, et al. Differentiating the pathologies of cerebral malaria by postmortem parasite counts. Nat Med 2004;10:143-5.

15 Mathers CD, Boerma T, Ma Fat D. Global and regional causes of death. Br Med Bull 2009;92:7-32.

16 World Health Organization (WHO). A standard verbal autopsy method for investigating causes of death in infants and children. Available: https://apps.who.int/iris/bitstream/handle/10665/ 66081/WHO_CDS_CSR_ISR_99.4.pdf?sequence=1\&isAllowed=y [Accessed 27 May 2020].

17 Desai M, ter Kuile FO, Nosten F, et al. Epidemiology and burden of malaria in pregnancy. Lancet Infect Dis 2007;7:93-104.

18 Castillo P, Ussene E, Ismail MR, et al. Pathological methods applied to the investigation of causes of death in developing countries: minimally invasive autopsy approach. PLoS One 2015;10:e0132057.

19 Martínez MJ, Massora S, Mandomando I, et al. Infectious cause of death determination using minimally invasive autopsies in developing countries. Diagn Microbiol Infect Dis 2016;84:80-6.

20 Maixenchs M, Anselmo R, Zielinski-Gutiérrez E, et al. Willingness to know the cause of death and hypothetical acceptability of the minimally invasive autopsy in six diverse African and Asian settings: a mixed methods Socio-Behavioural study. PLoS Med 2016;13:e1002172.

21 Rakislova N, Fernandes F, Lovane L, et al. Standardization of minimally invasive tissue sampling specimen collection and pathology training for the child health and mortality prevention surveillance network. Clin Infect Dis 2019;69:S302-10.

22 Bassat Q, Ordi J, Vila J, et al. Development of a post-mortem procedure to reduce the uncertainty regarding causes of death in developing countries. Lancet Glob Health 2013;1:e125-6.

23 Castillo P, Martínez MJ, Ussene E, et al. Validity of a minimally invasive autopsy for cause of death determination in adults in Mozambique: an observational study. PLoS Med 2016;13:e1002171.

24 Castillo P, Hurtado JC, Martínez MJ, et al. Validity of a minimally invasive autopsy for cause of death determination in maternal deaths in Mozambique: an observational study. PLoS Med 2017;14:e1002431.

25 Menendez C, Castillo P, Martínez MJ, et al. Validity of a minimally invasive autopsy for cause of death determination in stillborn babies and neonates in Mozambique: an observational study. PLoS Med 2017; 14:e1002318.

26 Bassat Q, Castillo P, Martínez MJ, et al. Validity of a minimally invasive autopsy tool for cause of death determination in pediatric deaths in Mozambique: an observational study. PLoS Med 2017; 14:e1002317.

27 Palhares AEM, Ferreira L, Freire M, et al. Performance of the minimally invasive autopsy tool for cause of death determination in adult deaths from the Brazilian Amazon: an observational study. Virchows Arch 2019;475:649-58.

28 Fernandes F, Castillo P, Bassat Q, et al. Contribution of the clinical information to the accuracy of the minimally invasive and the complete diagnostic autopsy. Hum Pathol 2019;85:184-93.

29 Saute F, Menendez C, Mayor A, et al. Malaria in pregnancy in rural Mozambique: the role of parity, submicroscopic and multiple Plasmodium falciparum infections. Trop Med Int Health 2002;7:19-28.

$30 \mathrm{WHO}$. The who application of ICD-10 to deaths during pregnancy, childbirth and puerperium: ICD MM, 2016. Available: http://www. who.int/reproductivehealth/publications/monitoring/9789241548458/ en [Accessed 27 May 2020].

31 Leitao J, Chandramohan D, Byass P, et al. Revising the who verbal autopsy instrument to facilitate routine cause-of-death monitoring Glob Health Action 2013;6:21518.

32 Byass P, Herbst K, Fottrell E, et al. Comparing verbal autopsy cause of death findings as determined by physician coding and probabilistic modelling: a public health analysis of 54000 deaths in Africa and Asia. J Glob Health 2015;5:010402.

33 Fottrell E, Byass P. Verbal autopsy: methods in transition. Epidemiol Rev 2010;32:38-55.
34 Byass P, Chandramohan D, Clark SJ, et al. Strengthening standardised interpretation of verbal autopsy data: the new InterVA-4 tool. Glob Health Action 2012;5:19281-8.

35 Fottrell E, Byass P, Ouedraogo TW, et al. Revealing the burden of maternal mortality: a probabilistic model for determining pregnancyrelated causes of death from verbal autopsies. Popul Health Metr 2007:5:1.

36 World Health Organization. Verbal autopsy standards: ascertaining and attributing causes of death. Available: https://www.who.int/ healthinfo/statistics/verbalautopsystandards/en/ [Accessed 28 May 2020]

37 ZR L, Mccormick TH, Clark SJ. InterVA4: an R package to analyze verbal autopsy data, 2014. Available: http://www.interva.net [Accessed 27 May 2020].

38 Taylor SM, Mayor A, Mombo-Ngoma G, et al. A quality control program within a clinical trial consortium for PCR protocols to detect plasmodium species. J Clin Microbiol 2014;52:2144-9.

39 Mayor A, Serra-Casas E, Bardaji A, et al. Sub-microscopic infections and long-term recrudescence of Plasmodium falciparum in Mozambican pregnant women. Malar J 2009;8:9

40 Kamau A, Mtanje G, Mataza C, et al. Malaria infection, disease and mortality among children and adults on the coast of Kenya. Malar $J$ 2020;19:210.

41 Ponsford MJ, Medana IM, Prapansilp P, et al. Sequestration and microvascular congestion are associated with coma in human cerebral malaria. J Infect Dis 2012;205:663-71.

42 Castillo P, Menéndez C, Mayor A, et al. Massive Plasmodium falciparum visceral sequestration: a cause of maternal death in Africa. Clin Microbiol Infect 2013;19:1035-41.

$43 \mathrm{Ye}$ Y, Kyobutungi C, Ogutu B, et al. Malaria mortality estimates: need for agreeable approach. Trop Med Int Health 2013;18:219-21.

44 Abdullah S, Adazu K, Masanja H, et al. Patterns of age-specific mortality in children in endemic areas of sub-Saharan Africa. Am J Trop Med Hyg 2007;77:99-105.

45 Deressa W, Fantahun M, Ali A. Malaria-related mortality based on verbal autopsy in an area of low endemicity in a predominantly rural population in Ethiopia. Malar $J$ 2007;6.

46 Snow RW, Armstrong JR, Forster D, et al. Childhood deaths in Africa: uses and limitations of verbal autopsies. Lancet 1992;340:351-5.

47 Selemani M, Mrema S, Shamte A, et al. Spatial and space-time clustering of mortality due to malaria in rural Tanzania: evidence from Ifakara and Rufiji health and demographic surveillance system sites. Malar J 2015;14:369.

48 Lozano R, Lopez AD, Atkinson C, et al. Performance of physiciancertified verbal autopsies: multisite validation study using clinical diagnostic gold standards. Popul Health Metr 2011;9.

49 Murray CJL, Ortblad KF, Guinovart C, et al. Global, regional, and national incidence and mortality for HIV, tuberculosis, and malaria during 1990-2013: a systematic analysis for the global burden of disease study 2013. Lancet 2014;384:1005-70.

50 Sacarlal J, Nhacolo AQ, Sigaúque B, et al. A 10 year study of the cause of death in children under 15 years in Manhiça, Mozambique. BMC Public Health 2009;9:67.

51 Desai M, Buff AM, Khagayi S, et al. Age-Specific malaria mortality rates in the KEMRI/CDC health and demographic surveillance system in Western Kenya, 2003-2010. PLoS One 2014;9:e106197.

52 Menéndez C, Romagosa C, Ismail MR, et al. An autopsy study of maternal mortality in Mozambique: the contribution of infectious diseases. PLoS Med 2008:5:e44.

53 Challe DP, Kamugisha ML, Mmbando BP, et al. Pattern of all-causes and cause-specific mortality in an area with progressively declining malaria burden in Korogwe district, north-eastern Tanzania. Malar $J$ 2018;17:97

54 Ngo Um Sap S, Tchaptchet K, Mekone I, et al. Mortality of children aged 5-15 years in a tertiary care center in Yaoundé, Cameroon. Arch Pediatr 2020;27:257-60.

55 Dhingra N, Jha P, Sharma VP, et al. Adult and child malaria mortality in India: a nationally representative mortality survey. Lancet 2010;376:1768-74

56 Kim Streatfield P, Khan WA, Bhuiya A, et al. Adult non-communicable disease mortality in Africa and Asia: evidence from indepth health and demographic surveillance system sites. Glob Health Action 2014;7:25365.

57 Menéndez C, Quintó L, Castillo P, et al. Limitations to current methods to estimate cause of death: a validation study of a verbal autopsy model. Gates Open Res 2020;4:55. 